\title{
Research on medical image retrieval algorithm
}

\author{
Wang Jinguo ${ }^{1, a}$, Wang $\mathrm{Na}^{2, b^{*}}$ (corresponding author), Wang rui ${ }^{3, c}$ \\ ${ }^{1}$ Department of Urology, the First Hospital of Jilin University, China \\ ${ }^{2}$ Department of Anesthesiology, the First Hospital of Jilin University, China \\ ${ }^{3}$ Department of Anesthesiology, the First Hospital of Jilin University, China \\ âwangjinguolily@163.com, ${ }^{\mathrm{b}}$ lilyly12345@163.com, ${ }^{\mathrm{C} X i a o b e n 6666 @ 126 . c o m ~}$
}

Keywords: Medical image. Retrieval algorithm. CBIR.

Abstract. Along with the development of medical imaging technology and the popularization of hospital information network (such as PACS, HIS, RIS), a large number of medical images which contain the physiological, pathological and anatomical information in a modern hospital every day will be produced. These images are an important objective basis for clinical diagnosis, disease tracking, surgical planning, study and differential diagnosis of the doctors. The complexity and importance of medical image data need to be highly effective in medical image retrieval and management methods, in order to maximize the use of medical images provided by the information.

\section{Introduction}

Along with the development of medical imaging technology and the popularization of hospital information network (such as PACS, HIS, RIS), a large number of medical images which contain the physiological, pathological and anatomical information in a modern hospital every day will be produced. These images are an important objective basis for clinical diagnosis, disease tracking, surgical planning, study and differential diagnosis of the doctors[1][2]. The complexity and importance of medical image data need to be highly effective in medical image retrieval and management methods, in order to maximize the use of medical images provided by the information. The most difficult problem in the retrieval of text is that it requires the doctors to extract medical images. And there are many drawbacks of manual annotation[3].

Based on text retrieval on a problem is the retrieval range has great limitations, such as can only retrieve a patient or a physician reports corresponding to the medical image, and in different patients, different physicians reported but there is disease of the same image retrieval is powerless, which in the field of differential diagnosis, medical statistics medical education and greatly limits the application of medical image. Image processing technology is used to extract gray, shape, texture, space and other characteristics parameters, which constitute the feature vector of image content, and feature vector as an objective basis to establish index and matching criterion. So it can be directly based on the feature vector of medical image, which is content-based image retrieval (CBIR)[4][5].

CBIR technology has avoided the trouble of text annotation, and extended the application of medical images in medical field[6]. It is an effective method for image retrieval, which is a new technology in the field of digital library, distributed image database, multimedia database, web search engine design and so on. Its successful application in the medical field will have a positive and far-reaching impact on clinical, medical research, telemedicine, remote consultation and medical education. 


\section{Content-based image retrieval}

In retrieval principle, both based on text image retrieval and content based image retrieval, mainly including three aspects: on the one hand the user needs the analysis and transformation of form can be retrieved from the index database of questions; on the other hand, collect and process the image resources, feature extraction, analysis and indexing, establish the index of the image database. Finally, on the one hand is according to the algorithm of the similarity calculated users ask and index database recorded in the similarity of the size and extraction to meet the recorded threshold as a result, according to the similarity in descending order of output[7].

In order to further improve the retrieval accuracy, many systems with relevance feedback technology to collect user feedback information on the retrieval results, which in CBIR is more prominent, because CBIR is stepwise refinement of the image retrieval process, need to constantly interacts with the user in the same retrieval process.Content based image retrieval is based on the content of the image and the context of the search, the image features of the image as a clue to detect other images with similar characteristics from the image database. Because the size of the image is generally greater than the pure text information, so the content based image retrieval is more efficient and more efficient.

The image input process will be input into the system, similar to the text content of the text retrieval system. System generally allows users to automatically or semi automatically (need user intervention) approach to image segmentation, identify the need for the object or content key points, so that the target feature extraction.

Feature extraction processing for the user or system marked by the image objects. Feature extraction can be done by human, for example, some of the key words can be described, and the corresponding image processing procedures can be done.

Some image features that are likely to be concerned with the retrieval of users. Feature extraction can is global, such as the color of the whole image distribution can also be according to local objects of an internal, such as image in the sub region. The feature representation method has many features such as color histogram, color moment, color set, etc., the texture representation method has Tamura texture features and texture features based on wavelet transform. However, when a feature is involved in the high level of image, it will be restricted by the knowledge domain and the search task.

\section{Performance evaluation of image retrieval}

Content based image retrieval uses a variety of different image features and similarity algorithm. For a specific image database, we need to choose one or more of the most effective image features and similarity algorithm. This requires a comprehensive evaluation of the effect of different conditions, and to find out the best way to compare different methods. In order to determine the performance of the retrieval system and the results obtained by different techniques, some measurement criteria are needed. Visual information retrieval is an interactive process, so the following performance index values are considered.

Recall and precision. Information retrieval is the process of comprehensive query, description, matching and extraction. Based on the principle of pattern recognition, is related to the correct retrieval, undetected, not associated with the error detection and correct rejections 4, on retrieval evaluation that the retrieval result is correct or not, mainly using the two indicators of precision and recall. The meaning of precision and is in a query, the system returns the query results in image 
number of accounts for all return in proportion to the number of images; recall refers to the number of returns to the system results in the image for image library, all related to the number of images than cases. Users in the evaluation of the results of the query can be determined in advance some of the images as relevant to the query image and according to the results returned by the system to calculate precision and recall. The higher the value of these two indicators, the better the effect of the method.

Effectiveness. To measurement system of recall and accuracy, often need to thousands of sample statistics to get good results, and the validity of the measurement system requires only a small image of the group. These images include visual elements that are not identical to the query graph, but they are also similar to the query graph. In the experiment, the system can be used to evaluate the function of the system. Specific experiments in each image $\mathrm{i}$ and each retrieval example $\mathrm{j}$, determine a system to the distribution of the order $P_{j}(i)$ as the center, the width of the window $\sigma_{j}(i)$. The effectiveness of the retrieval is inferred by comparing the system and the ranking of the image $\mathrm{i}$ with respect to the query example $\mathrm{j}$. A sort of measurement can be expressed as:

$$
S_{j}(i)=\sum_{k=P_{j}(i)-\sigma_{j}(i)}^{P_{j}(i)+\sigma_{j}(i)} Q_{j}(i, k)
$$

The measure gives the sum of $Q_{j}(i)$ values by the image i between the position $P_{j}(i)-\sigma_{j}(i)$ and $P_{j}(i)+\sigma_{j}(i)$ and according to measure value, we can compare the retrieval performances.

efficiency of retrieval. To a given query image, let $\mathrm{N}$ be the total number of similar images in the image library, $\mathrm{n}$ is extracted from the image number, $\mathrm{T}$ is the system extracts the sum of the image, then the retrieval efficiency is defined as:

$$
\eta_{T}= \begin{cases}n / N & N<T \\ n / T & N>T\end{cases}
$$

When a series of experiments are carried out, the total number of images can be extracted, so that all the similar images can be retrieved and the correct order of the images is determined.

Retrieval ratio. For a given query image, the extracted image should be a function of the retrieval technique parameters. This can be defined as an excellent criterion for the retrieval rate. For a specific search technology, when given a query plan for a given set of parameters, the retrieval rate can be given a set of parameters:

$$
\eta_{i j}(N, T)=\frac{n_{i j}}{N} \quad i=1,2, \ldots ., M \quad j=1,2, \ldots \ldots, K
$$

Where $\mathrm{M}$ is the total number of query images, $\mathrm{K}$ is the total number of parameters collection, $\mathrm{N}$ and $\mathrm{T}$ of the meaning and retrieval efficiency of the same. 


\section{References}

[1] W Niblack,R Barber,W Equitz,et al.The QBIC Project: Querying Image by Content Using Color, Texture, and Shape. Proceedings of the Academy of Natural Sciences of Philadelphia . 1993.

[2] Rui Y,Huang T S,Mehrotra S.Content -based image retrieval with relevance feedback in MARS. Proceedings of the IEEE International Conference on Image Processing . 1997.

[3] John R Smith,Shih-Fu Chang.VisualSEEK: a fully automated content-based image query system. Proceedings of the Fourth ACM Multimedia Conference (MULTIMEDIA 96) . 1996.

[4] Carson C,Thomas M,Belongie S,et al.Blobworld: a system for region-based image indexing and retrieval. Proc Third International Conference on Visual Information Systems . 1999.

[5] Bach JR,Fuller C,Gupta A,et al.The Virage image search engine: An open framework for image management. Proceedings of the SPIE,Storage and Retrieval for Image and Video Databases IV . 1996.

[6] Rui, Yong,Huang, Thomas S.,Ortega, Michael,Mehrotra, Sharad.Relevance feedback: A power tool for interactive content-based image retrieval. IEEE Transactions on Circuits and Systems for Video Technology . 1998.

[7] Jie Gui,Zhenan Sun,Wei Jia,Rongxiang Hu,Yingke Lei,Shuiwang Ji. Discriminant sparse neighborhood preserving embedding for face recognition[J]. Pattern Recognition . 2012 (8). 